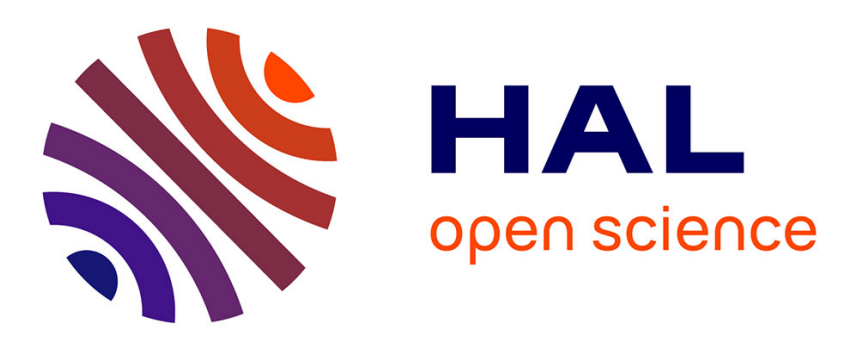

\title{
Characterization of MEMS resonators via feedthrough de-embedding of harmonic and subharmonic pulsed-mode response
}

Alexis Brenes, Jérôme Juillard, Filipe Vinci dos Santos, Alain Bonnoit

\section{- To cite this version:}

Alexis Brenes, Jérôme Juillard, Filipe Vinci dos Santos, Alain Bonnoit. Characterization of MEMS resonators via feedthrough de-embedding of harmonic and subharmonic pulsed-mode response. Sensors and Actuators A: Physical , 2015, 229, pp.211-217. 10.1016/j.sna.2015.02.032 . hal-01235587

HAL Id: hal-01235587

https://hal-centralesupelec.archives-ouvertes.fr/hal-01235587

Submitted on 1 Dec 2015

HAL is a multi-disciplinary open access archive for the deposit and dissemination of scientific research documents, whether they are published or not. The documents may come from teaching and research institutions in France or abroad, or from public or private research centers.
L'archive ouverte pluridisciplinaire HAL, est destinée au dépôt et à la diffusion de documents scientifiques de niveau recherche, publiés ou non, émanant des établissements d'enseignement et de recherche français ou étrangers, des laboratoires publics ou privés. 


\title{
Characterization of MEMS resonators via feedthrough de- embedding of harmonic and subharmonic pulsed-mode response
}

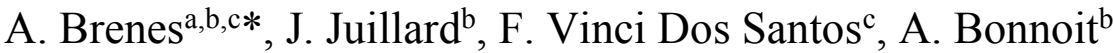 \\ ${ }^{a}$ THALES Avionics SAS, 25 rue Jules Védrines, 26000 Valence, France \\ ${ }^{b}$ SSE, Ecole Supérieure d'Electricité, 3, rue Joliot-Curie, 91192 Gif-sur-Yvette, France \\ ${ }^{c}$ THALES Chair, Ecole Supérieure d'Electricité, 3, rue Joliot-Curie, 91192 Gif-sur-Yvette, France
}

\begin{abstract}
In this paper, we model and give experimental evidence of the efficiency of pulsed-mode actuation for the characterization of capacitive MEMS resonators. In particular, we show how to process the pulsed-mode response in two modes (harmonic and subharmonic) so as to eliminate parasitic feedthrough effects and characterize the resonator more accurately. We test our approach on a pressure sensor, developed by THALES Avionics, used for avionics applications. In contrast to the existing stateof-the-art techniques, the methods described in this paper can be used to characterize electrostatic MEMS without the use of a spectrum analyzer or high-performance ADC.
\end{abstract}

Keywords: Capacitive feedthrough; MEMS characterization; open-loop actuation; pulsed-mode response; time domain signal processing.

\section{Introduction}

Feedthrough is a major obstacle to the characterization of capacitive MEMS resonators via electrical measurements [1-4]. Open-loop frequency responses are distorted by parasitic feedthrough, which leads to poor-quality estimations of the natural frequency and Q-factor of the resonator. Previous work has addressed direct parameter extraction from feedthrough-embedded frequency response via considerations on the Nyquist plot and the estimation of the parasitic capacitance [1]. Another option consists in de-embedding parasitic feedthrough via subharmonic actuation. This method usually requires a spectrum analyzer to extract the resonant signal from a large subharmonic signal [2,3], which is a drawback in embedded systems. Another class of methods is based on amplitude measurements via multisideband demodulation [4]. However, the method described in [4] also requires a precise spectral analysis of the output signals. Moreover, it is based on a precise model of the capacitive detection non-linearity. While such a model may be available in several applications, there are also many cases in which the exact expression of the nonlinearity is not known and the method cannot be applied.

Foregoing these spectral approaches, one time-domain characterization method for MEMS resonators has recently been introduced [5]. It consists in ring-down measurements and is based on the record of exponentially decaying amplitude This method is especially efficient for very high-Q, low-frequency resonators for which the previous methods based on steady-state regime measurements could take days and would require the generation of a very small and accurate actuation voltage. Interesting though it may be, it is sensitive to the number of zero-crossings to be measured, which is a drawback for high-Q resonators.

In this article, we present a new class of time-domain characterization methods robust to feedthrough effects that is simpler and free of the shortcomings of existing techniques. The principle of feedthrough de-embedding using pulsed-wave actuation and the experimental setup are described in section 2 . In section 3 , the method is illustrated and our experimental results are compared to those obtained with sine-wave actuation.

\footnotetext{
* Corresponding author. Tel.: +33-1-69-85-14-17.

E-mail address: alexis.brenes@centralesupelec.fr
} 


\section{Feedthrough de-embedding}

\subsection{Experimental setup}

The resonator experimentally characterized in this study was originally developed by SEXTANT Avionics (currently THALES) [6]. It is industrially assembled by the fusion-bonding of three etched silicon wafers (see Fig. 1a) and consists of a resonant beam resting on a rectangular diaphragm. During the manufacturing process, the beam is encapsulated in vacuum to achieve a high mechanical Q-factor $(\approx 20000)$. The resonance frequency $f_{0}$ of the device is close to $65 \mathrm{kHz}$.

(a)

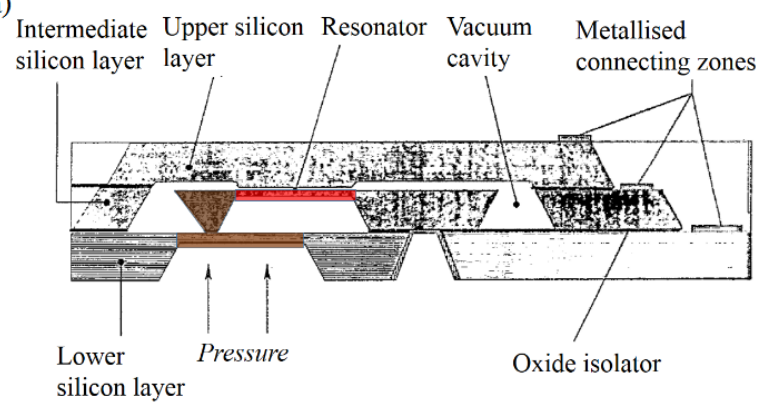

(b)

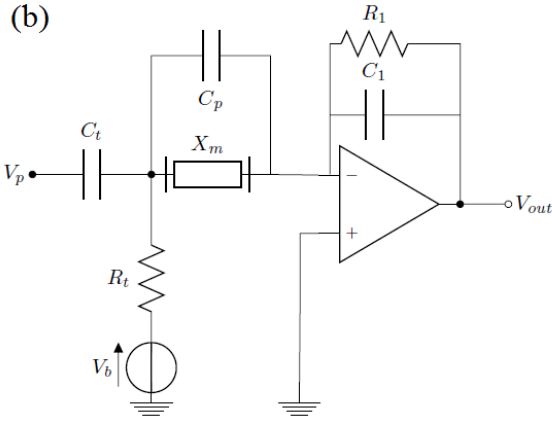

Fig. 1. (a) Sensor structure (from [6]). The sensing element consists of a resonant silicon beam encapsulated in vacuum, with a massive stud bonded to a silicon diaphragm. The beam is capacitively actuated with the facing electrode. (b) Electronic architecture. The Xm-element is the resonator. $C_{p}$ is the unknown parasitic capacitance responsible for feedthrough. $C_{t}=1 \mu F, R_{t}=1 \mathrm{k} \Omega, C_{1}=10 p F$ and $R_{1}=1.0 M \Omega$.

In our setup, a bias voltage $V_{b}$ is applied to the resonator. It is directly actuated in open-loop by a waveform generator delivering voltage pulses of width $T_{p}$ and amplitude $V_{\mathrm{p}}$, repeated every $T$ seconds, where $T(=1 / f)$ is close to $T_{0}=1 / f_{0}$. The motion of the resonator gives rise to a motional current which is integrated in a charge amplifier (see Fig. 1-b). Hence, the output voltage $V_{\text {out }}$ is the image of the mechanical motion of the resonator (slightly distorted by the capacitive detection nonlinearity). $V_{\text {out }}$ is recorded with an oscilloscope for every pulse frequency. Due to the parasitic feedthrough, unavoidable in this kind of capacitive actuation and detection scheme, voltage pulses are superposed on the motional signal (Fig. 2-a).
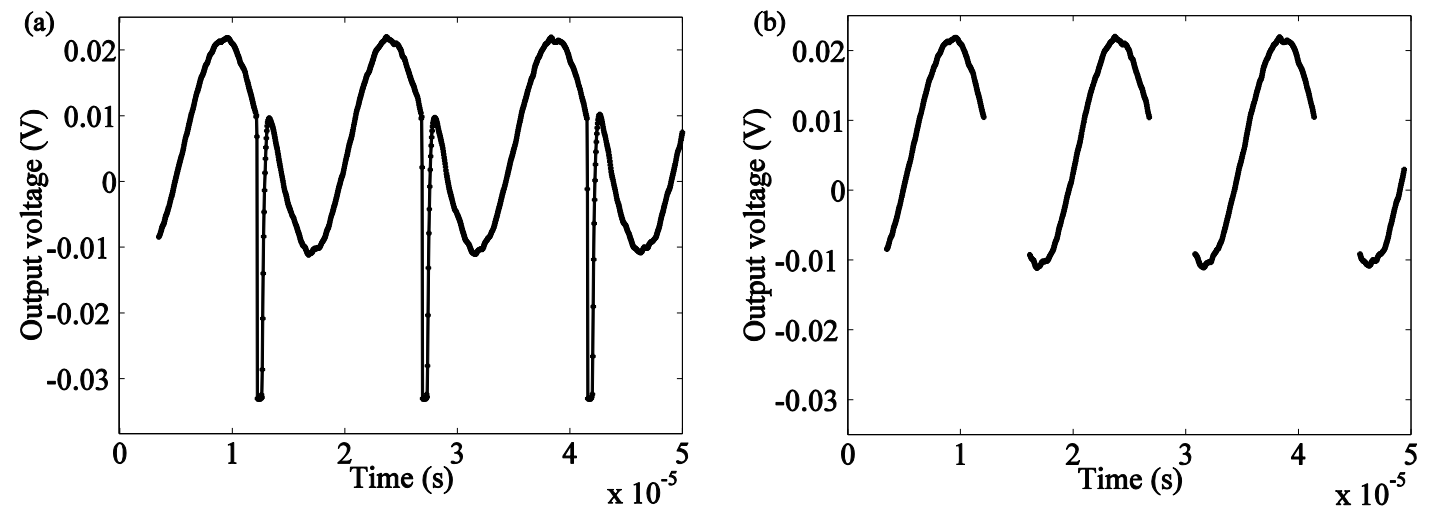

Fig. 2. Typical shape of the output voltage close to resonance with pulsed-mode actuation before (a) and after (b) pulse removal.

\subsection{Principle of feedthrough de-embedding by harmonic pulsed-mode actuation (HPMA)}

Looking at the output signal, one can observe that feedthrough distortion is concentrated within the pulses, i.e. 
localized in time. This is the key difference between pulsed-wave and sine-wave actuation. Thus, the pulses can be effortlessly located and removed from the waveform via post-processing (using Matlab, for example), as shown in Fig. 2-b. A $T$-periodic sine-wave can then be fitted (in the least-squares sense) to the cleaned-up signal, allowing one to extract the amplitude and phase of the resonator response $V_{\text {out }}$. Sweeping $T$ in the neighborhood of $T_{0}$ (Harmonic Pulsed-Mode Actuation), one may then obtain a frequency response similar to the one which would be obtained with sine-wave excitation, as explained in sub-section 2.4, but with most of the feedthrough effects cancelled.

\subsection{Principle of feedthrough de-embedding by subharmonic pulsed-mode actuation (SPMA)}

The accuracy of the proposed HPMA signal separation is sensitive to the shape, the amplitude and the width of the pulses going through the circuit. These not only depend on the capacitive feedthrough, but also on the cutoff frequency of the electronics and on the (a priori unknown) parasitic input and output impedances of the resonator. Consequently, the pulse may be distorted and spread over time, so that part of the motional signal remaining after pulse removal may still be polluted by the "tail" of the distorted pulse.

In order to overcome these obstacles, we propose to increase the period of the excitation signal, so that more than one period of the motional signal can be observed between two pulses. The resonance can then be observed clearly whenever the period $T$ is close to an integer multiple $N$ of $T_{0}$. In a nutshell, the larger $N$ is, the easier it is to distinguish the desired signal from the pulsed feedthrough signal. A typical measurement, acquired for $T$ close to $12 T_{0}$ (i.e. $N=12$ ), is shown in Fig.3-a. Using SPMA (or HPMA) and examining the shape of the output voltage, one can estimate at a glance the resonant frequency. Regardless of the shape of the pulses and of what distorts them, one may observe that the signal in the wake of any pulse is a very clean sine-wave (Fig. 3-b), which may then be recorded and processed. The only noteworthy artifact is a positive overshoot after each pulse which vanishes to zero after a few periods of the motional signal, and which is partly due to nonlinear effects in the op-amp. In order to apply these concepts for automated and reliable parameter extraction, we give next a mathematical derivation of the fit equations for each case.
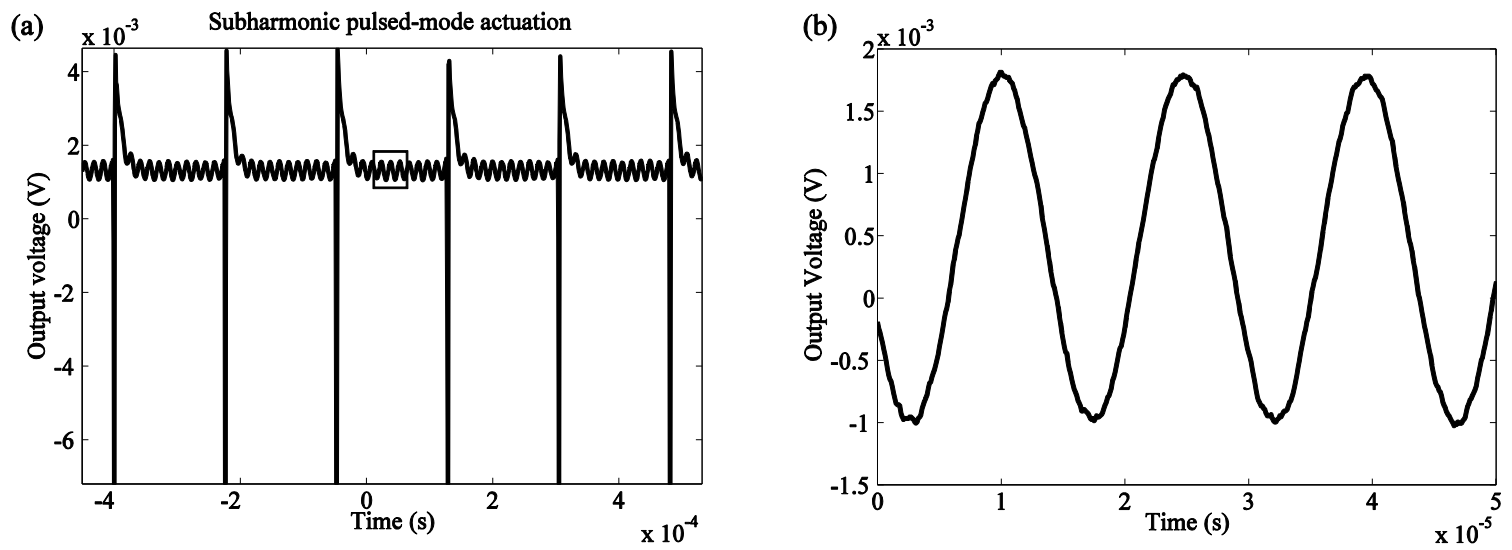

Fig. 3: Shape of the output voltage the pulses for $T \approx 12 T_{0}$ (a) and selection of the sine-wave temporally separated from feedthrough pulses (b).

\subsection{Determination of the pulsed-mode frequency response}

Assuming that the width $T_{p}$ of the pulses is very short compared to $T$, the output of the resonator over one period may be written as the response of a $2^{\text {nd }}$ order linear system with static gain $G$ to an impulse train (Dirac comb), i.e.

$$
s(t)=2 \pi G V_{p} T_{p} f \operatorname{Re}\left(\exp \left(\left(-\alpha+j 2 \pi f_{1}\right) t\right) \sum_{n=0}^{\infty} r^{n}\right) \text { where } r=\exp \left(\left(-\alpha+j 2 \pi f_{1}\right) T\right), \alpha=\frac{\pi f_{0}}{Q} \text { and } f_{1}=f_{0} \sqrt{1-\frac{1}{4 Q^{2}}} \text {. }
$$


Without limiting the general scope of the preceding relations, one may consider a pulse triggered at $t=0$ and $t$ in the interval $[0, T]$. Evaluating the limit of the geometric series leads to a general analytical expression of $s(t)$, under the assumption $Q \gg>2 \pi$.

$$
s(t) \approx \frac{2 \pi V_{p} T_{p} f}{\sqrt{1+\exp \left(-\frac{2 \alpha}{f}\right)-2 \exp \left(-\frac{\alpha}{f}\right) \cos \left(\frac{2 \pi f_{1}}{f}\right)}} \sin \left(2 \pi f_{1} t+\Phi_{p}\right) \text { with } \Phi_{p}=\arctan \left(\frac{\exp \left(-\frac{\alpha}{f}\right) \cos \left(\frac{2 \pi f_{1}}{f}\right)-1}{\exp \left(-\frac{\alpha}{f}\right) \sin \left(\frac{2 \pi f_{1}}{f}\right)}\right) .(1)
$$

From now on, we limit ourselves to $2 \pi|X|<<1$, where $X=f_{0} / f-1$. Second-order Taylor expansions about the point $(X, 2 \pi / Q)=(0,0)$ yield:

$$
s(t) \approx S \sin \left(2 \pi f t+\Phi_{p}\right) \text { with } S=2 V_{p} T_{p} f_{0} \frac{G Q}{\sqrt{1+4 Q^{2} X^{2}}} \text { and } \Phi_{p}=\arctan (2 Q X)-\frac{\pi}{2}
$$

Thus, given these assumptions, the amplitude and phase response obtained with HPMA are similar to those obtained with sine-wave actuation, provided the sine-wave amplitude $V_{\text {sin }}$ equals $2 V_{p} T_{p} f_{0}$.

The relative error on amplitude between (2) and (1) is plotted in Fig. 4-a. For a quality factor estimated at 20000, a reasonable sweep range to determine its precise value is for instance $\left[(1-\mathrm{p}) f_{0} ;(1+\mathrm{p}) f_{0}\right]$ where $\mathrm{p}=2.10^{-4}$. In this range, the maximal relative error is less than $0.01 \%$ (see Fig. 4 -a.)

\subsection{Determination of the subharmonic pulsed-mode frequency response}

If one considers subharmonic actuation (SPMA), then $T \approx N T_{0}(N \in \mathbb{N} \backslash\{0\})$, a different set of assumptions $(Q>>2 \pi N$ and $2 \pi N|X|<<1)$ combined with similar mathematical developments as given in sub-section 2.4. yield:

$$
S=\frac{2 V_{p} T_{p} f_{0}}{N} \frac{G Q}{\sqrt{1+4 Q^{2} X^{2}}} \text { and } \Phi_{p}=\arctan (2 Q X)-\frac{\pi}{2} .
$$

Thus, one obtains a relationship equivalent to (2), but with an amplitude dependency on $N$.

Fig 4-b depicts the relative error on amplitude between (3) and (1) divided by N. Considering the previous assumption $Q>>2 \pi N$, the relative error increases with $N$. Nevertheless, one can notice that, for a quality factor of 20000 , the difference between (3) and (1) is below $0.5 \%$ even for high values of $N$ (e.g. $N=50$ ). However, for lower quality factors, $N$ should be carefully chosen. 

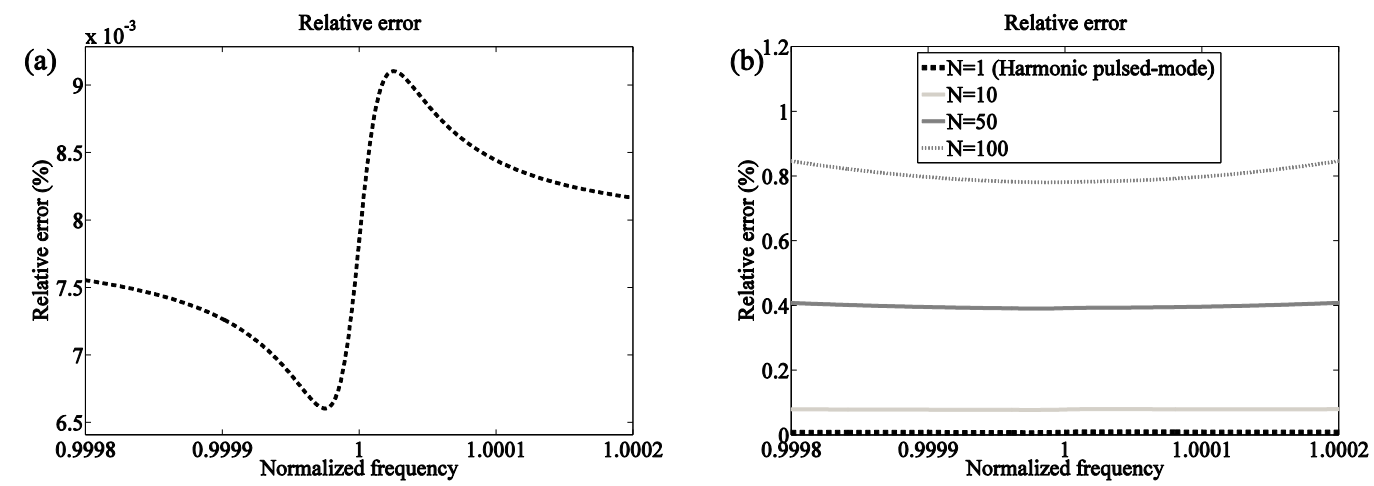

Fig. 4: (a) Relative error on amplitude with formula (2) $(Q=20000)$. (b) Relative error on amplitude with formula (3) for different values of $N$ $(Q=20000)$. The curve plotted in (a) corresponds to $N=1$ in (b).

\subsection{Experimental limit of subharmonic pulsed-mode actuation}

To improve subharmonic pulsed-mode measurements, one may want to increase the length of the recorded sine wave, i.e. choose $N$ arbitrarily high. However, the value of $N$ is limited by (i) the power available to the $f_{0}$-harmonic, (ii) the quality factor of the resonator, (iii) the finite duration of the excitation pulses.

(i) is illustrated by formula (3), as an increase of $\mathrm{N}$ reduces the power available to the resonator. To compensate for this loss, one must increase the pulse width, which experimentally limits the value of $N$.

To understand (ii), the assumption $N<<Q /(2 \pi)$ (see Fig. 4-b) must be kept in mind. If one wants to overcome this limit, the more general expression (1) must be considered instead of (3).

To illustrate (iii), one must bear in mind that the duty-cycle of the pulse generator is technically limited, so that we are forced to increase the length of the pulses as $N$ grows. Yet, the length of the pulses is finite, as a consequence the spectral representation of the actuation voltage is not in reality a Dirac comb, but has instead a cardinal-sine low-pass characteristic, as depicted in Fig. 5. This is the main experimental limit to $N$. A balance must be struck between, on the one hand, increasing $N$ (as in Fig. 3) and, on the other hand, maintaining enough amplitude on the $f_{0}$-harmonic of the actuation voltage. The first requires a large value of $N$ while the latter limits this value. Practically speaking, we observe resonance for high values of $N(N \leq 100)$. However, for larger values of $N$, the SNR of the output voltage $V_{\text {out }}$ is insufficient to exploit measurements and may even reach zero for a very large $N$ (see illustration $\mathrm{N}=48$ in Fig. 5).
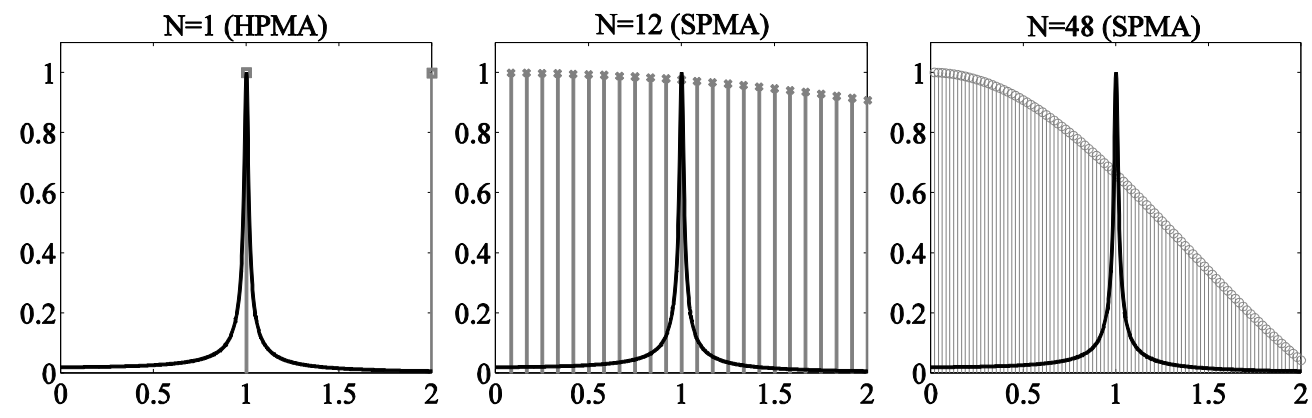

Fig. 5. Illustration of subharmonic actuation for a resonator with $\mathrm{N}=1$ (left), $\mathrm{N}=12$ (center) or $\mathrm{N}=48$ (right). The vertical lines represent the actuation Dirac comb. The black line illustrates the bode diagram of the resonator. Experimentally, only several harmonics compose the Dirac comb because of its cardinal-sine envelope. 


\section{Results}

\subsection{Simulations}

In this section, we report the simulated frequency responses obtained in the presence of capacitive feedthrough for the following three methods: (i) sine-wave actuation $\left(V_{\sin }=39 \mathrm{mV}\right)$, (ii) harmonic pulsed-wave actuation $\left(V_{p}=2 \mathrm{~V}\right.$, $T_{p}=150 \mathrm{~ns}$ ) without signal post-processing (as performed in [5]), and (iii) with partial or (iv) total pulse-removal processing. The comparison between the two last cases helps to evaluate the impact of imperfect data processing, which is the main drawback of our method. Since the use of SPMA instead of HPMA has no influence on the normalized frequency response (see (2) and (3)), we do not report the results of simulations obtained with SPMA.

The typical shape of sine-wave and pulsed-mode actuation voltages are reported in Fig. 6 and the corresponding frequency responses in Fig. 7. For each method, the estimated amplitude and phase are obtained through least-squares fitting of a sine-wave with known frequency $f$ over 3 periods of the simulated signal, sampled at $f_{s}>1 / T_{\mathrm{p}}$. Thus, in methods (i) and (ii) a total of $3 T f_{s}$ points are used for fitting. In method (iii), the signal is processed before performing the fit: the pulses are localized in time and the feedthrough pulse data points are removed, leaving $3\left(T-K T_{p}\right) f_{s}$ points to perform the fit, where $K \leq 1$ is a user-chosen parameter, illustrating the effect of partially or totally cleaning out the signal (i.e. $K=1$ corresponds to (iv)). Here, we chose $K=0.98$ to illustrate (iii). The Bode diagrams obtained with method (i) and (ii) perfectly coincide. Feedthrough is greatly reduced with partial pulse removal and is completely cancelled with total pulse removal.

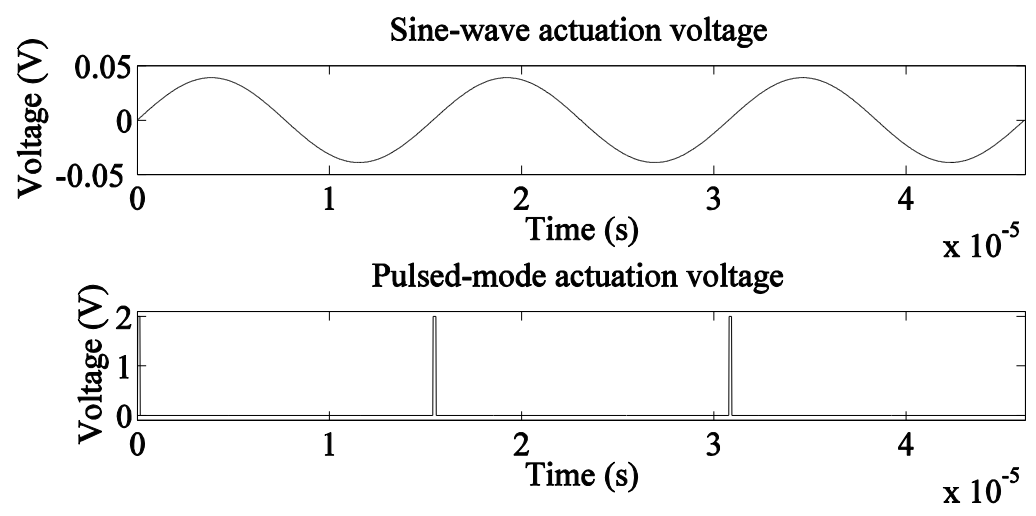

Fig. 6. Shape of typical simulated actuation voltages: Simulated sine-wave actuation and equivalent harmonic pulsed-mode actuation $\left(V_{\sin }=2 V_{p} T_{p} f_{0}\right)$.
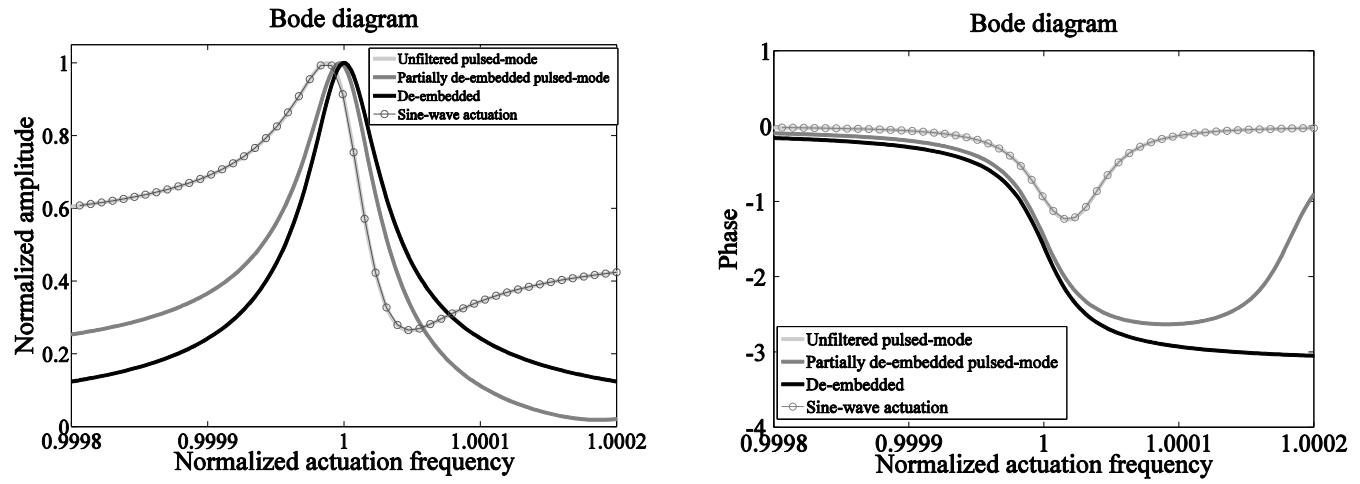

Fig. 7. Simulated Bode diagrams obtained with methods (i) and (ii) (light-grey line and circle line), (iii) (dark-grey) and (iv) (dark). 


\subsection{Experimental results with harmonic pulsed-mode actuation (HPMA)}

Experimental results obtained with harmonic pulsed-mode actuation are shown in Fig. 8. There is a good agreement between the experimental results and those predicted by the simulations, except one must choose $K>1$ (here $K=30$, as represented in Fig. 2) in method (iii) to perfectly cancel the effect of feedthrough as explained in sub-section 2.3. The estimated quality factor and natural frequency are respectively 21000 and $68.1 \mathrm{kHz}$, close to the expected values. However, the fit between the experimental and theoretical frequency response is not perfect, mostly because of imperfect feedthrough cancellation, as shown in the next sub-section. The comparison with a more accurate estimation of the quality factor is made in sub-section 3.3.
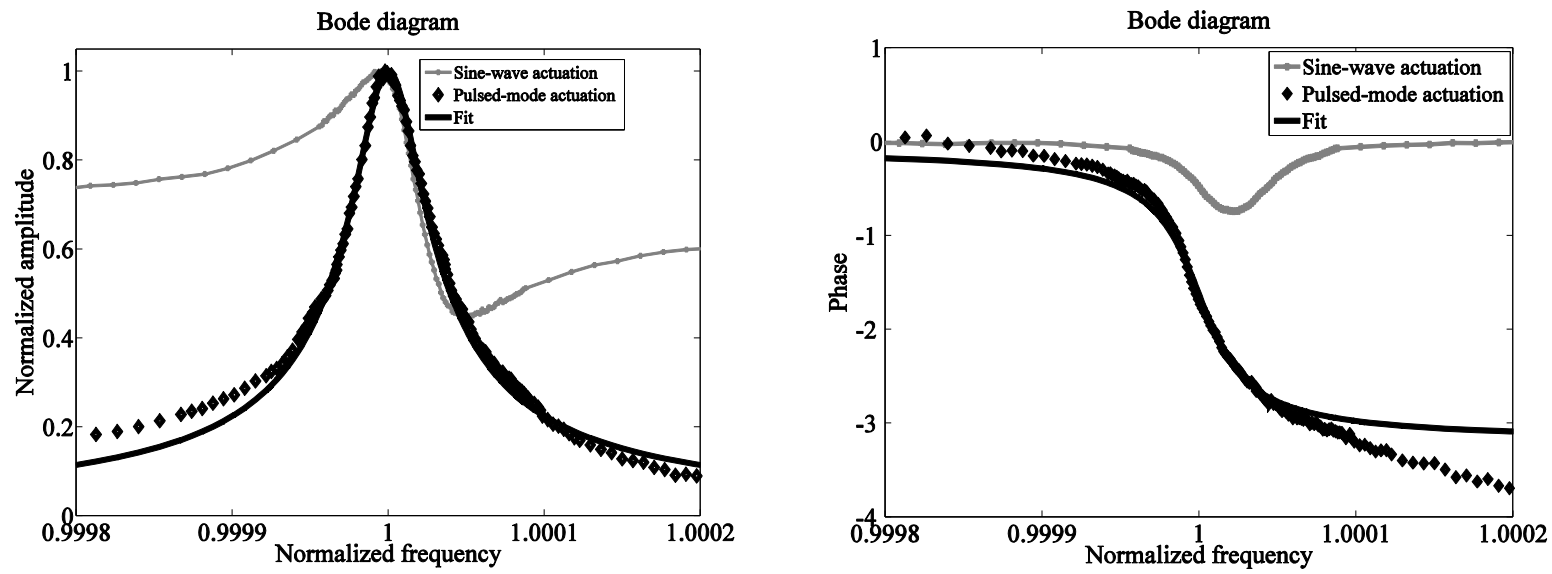

Fig. 8. Experimental Bode diagrams obtained with method (i) (grey crosses) and method (iii) (diamonds). The continuous dark line shows the curve fitted from (3) to the measured amplitude and phase obtained with method (iii).

\subsection{Experimental results with subharmonic pulsed-mode actuation (SPMA)}

Experimental results obtained with subharmonic pulsed-mode actuation are reported in Fig. 9. In this experimental setup, $T$ has been swept around $12 T_{0}(N=12)$. As highlighted in Fig. 9, the fit is extremely precise and absolutely no feedthough embedding is observed. This method gives $Q=18900$ and $f_{0}=68.0 \mathrm{kHz}$, close to the expected values.
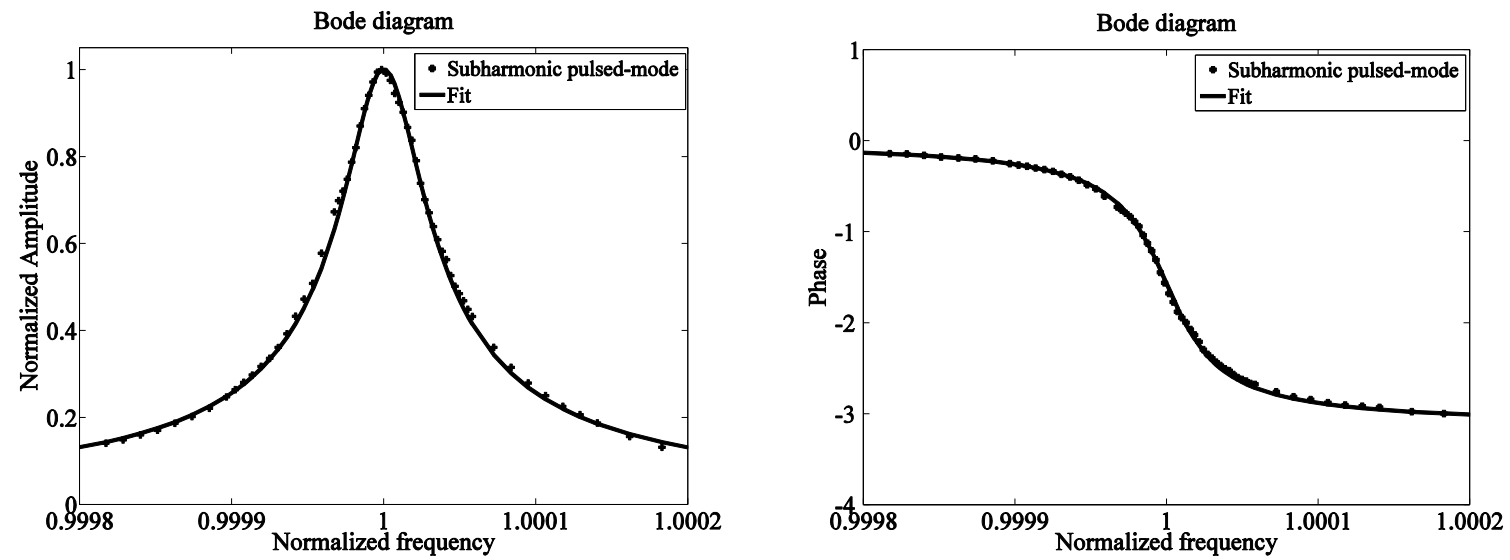

Fig. 9. Bode diagram obtained with subharmonic pulsed-mode actuation. The continuous dark line shows the curve fitted to this response. 
The value of $Q=18900$ obtained with SPMA underlines that HPMA overestimated the quality factor by $11 \%$. An explanation for this overestimation is given in sub-section 3.4. Despite this error, HPMA remains an interesting method since it reduces the risk of actuating unwanted resonance modes with natural frequency within the sweep range.

\subsection{Comparison between de-embedded harmonic pulsed-mode actuation and subharmonic pulsed-mode actuation}

For each set of measurements of Fig. 8 and Fig. 9, we report the cumulative mean squared error (CMSE) between the fitted curves and the experimental responses in Fig. 9. CMSE has been computed with (4), where $x_{m e s}$ and $x_{f i t}$ respectively correspond to the experimental and the fitted curves, for a sweep between $1-f_{\text {end }}$ and $1+f_{\text {end }}$ (here $f_{\text {end }}=0.0002$ ).

$$
\varepsilon(f)=\sqrt{\frac{\sum_{1-f}^{1+f}\left(x_{\text {mes }}(f)-x_{f i t}(f)\right)^{2} \Delta f}{\mu}} \text { where } \mu=\sum_{1-f_{\text {end }}}^{1-f_{\text {end }}} x_{f i t}(f)^{2} \Delta f .
$$

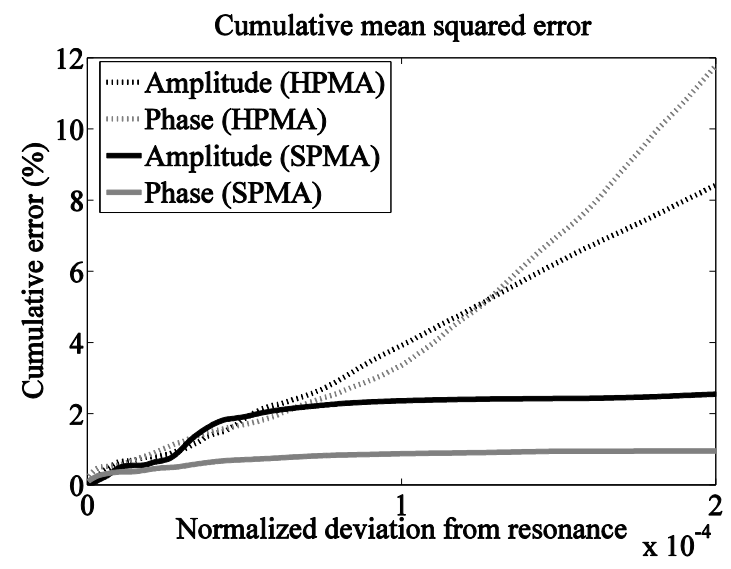

Fig. 10. Cumulative mean squared error computed with (4) between the fitted and experimental curves for HPMA (dotted diamond lines) and SPMA (solid crossed lines).

As Fig. 10 highlights, the total cumulative error is $2.6 \%$ on amplitude and $1 \%$ on phase with SPMA, whereas it is close to $10 \%$ with HPMA on both amplitude and phase. The HPMA error is mostly related to the poorer precision far from resonance, which is explained by the degraded sine-wave fit obtained with low-amplitude truncated signals. To compensate for this effect, one has to increase the number of measurements close to resonance (as in Fig. 8 compared to Fig. 9). Otherwise, the lorentzian fit becomes uncertain, nay impossible.

The higher error on HPMA may explain the 11\%-overestimation of the quality factor induced by HPMA in this setup (see sub-section 3.3), even though this overestimation may also be due to slight changes in the experimental conditions (e.g. temperature or pressure) between the two sets of measurements.

The remaining error with SPMA may be explained by low-amplitude noise on electrical measures. Since this resonator is already known to exhibit parametric resonance under certain circumstances [7], we also suspect some of the discrepancies to be related to similar phenomena.

\section{Conclusion}

The original ideas and measurements presented in this paper show that pulsed-mode actuation of MEMS resonators allows improved frequency response analysis. We highlighted the similarities between the theoretical sine-wave and 
pulsed-mode frequency response for high-Q resonators and devised two different methods to extract a pulsed-mode response. The proposed approach is based on a simple post-processing of the measured signal, as opposed to existing methods based on semi-harmonic sine-wave actuation. Better results were obtained using subharmonic pulsed-mode actuation, but both methods introduced in this paper suppress greatly the parasitic feedthrough effects and permit a good characterization of the resonator, as validated through experiments on a resonant MEMS sensor. Interestingly, pulsed-mode actuation is adaptable to any high-Q MEMS device without specific architecture or sensing scheme, as opposed to technological solutions (e.g. piezoelectric sensing or differential measurements) [3,8]. Moreover, it does not require to measure the parasitic capacitance of the MEMS device which may be affected by long-term effects such as creep or fatigue $[9,10]$. Our method also suppresses the remaining capacitive feedthrough in pulsed-mode spectral measurements [11].

Further work will aim at automating the characterization procedure, eventually through analog signal processing. In a broader perspective, a relevant combination of pulsed-mode measurements might be an effective and robust methodology to characterize multiple resonant modes and parametric resonances.

\section{References}

[1] J. E.-Y. Lee, A. A. Seshia, Direct parameter extraction in feedthrough-embedded capacitive MEMS resonators, Sensors and Actuators A, 167 (2011), $237-244$.

[2] V.J. Logeeswaran, F.E.H. Tay, et al, First Harmonic (2f) Characterisation of Resonant Frequency and Q-Factor of Micromechanical Transducers, Analog Integrated Circuits and Signal Processing, 37 (2003), 17 - 33.

[3] A. T-H. Lin, J. E-Y. Lee, et al., Enhanced transduction methods for electrostatically driven MEMS resonators, Solid-State Sensors, Actuators and Microsystems Conference (TRANSDUCERS) (2009), 561 - 564.

[4] A. A. Trusov, A. M. Shkel, A Novel Capacitive Detection Scheme with Inherent Self-Calibration, IEEE/ASME Journal of Microelectromechanical Systems, 16, 6 (2007), 1324- 1333.

[5] Z. Zeng, M. A. P. Pertijs et al., An energy-efficient readout circuit for resonant sensors based on ring-down measurement, Review of Scientific Instruments, 84 (2013).

[6] J. Mandle, O. Lefort et al., A new micromachined silicon high-accuracy pressure sensor, Sensors and Actuators A, 46-47 (1995), 129 - 132.

[7] A. Brenes, J. Juillard et al., Electrostatically-induced modal crosstalk phenomena in resonant MEMS sensors, Proceedings of DTIP (2014), 294 $-297$.

[8] Y. Xu, J. E.-Y. Lee, Single-Device and On-Chip Feedthrough Cancellation for Hybrid MEMS Resonators, IEEE Transactions on Industrial Electronics, 59, 12 (2012), 4930 - 4937.

[9] R. Modlinski, A. Witvrouw et al., Creep as a reliability problem in MEMS, Microelectronics Reliability, 44 (2004), 1733 - 1738.

[10] T. Ando, M. Shikida et al., Tensile-mode fatigue testing of silicon films as structural materials for MEMS, Sensors and Actuators A, 93 (2001), $70-75$.

[11] J.L. Muñoz-Gamarra, E. Marigó et al, Characterization of CMOS-MEMS resonator by pulsed mode electrostatic actuation, Frequency Control Symposium (FCS) IEEE International, (2010) 415 - 418.

\section{Biographies}

Alexis Brenes was born in Brussels, Belgium, in 1987. He earned an Engineering degree from Ecole Centrale Paris (ECP), Châtenay-Malabry, France in 2010 and Master's degrees in Physics and Nanotechnology from Ecole Normale Supérieure (ENS), Cachan, France, in 2012 and 2013. He has been researching characterization methods and nonlinearities in MEMS since March 2013. He is currently a Ph.D. candidate at the Ecole Supérieure d'Electricité (Supélec), employed by THALES Avionics, under the supervision of Pr. J. Juillard and Pr. F. Vinci dos Santos. His research interests include sensors for aeronautics, MEMS characterization, nonlinear oscillators, design and testing.

Jérôme Juillard was born in Nice, France, in 1973. He graduated from the Ecole Centrale Paris (ECP) in 1995, specializing in electrical engineering. Following an M.Sc.in acoustics and signal processing at IRCAM, he joined the CEA and received his Ph.D. degree in physical acoustics from University Paris 7 in 1999. In 2000, he joined Supélec, where he is now a professor. His research interests include nonlinear oscillators, oscillator networks, as well as MEMS and NEMS modeling, design and testing.

Filipe Vinci Dos Santos earned his B.Sc. (electronics engineering) and M.Sc. (microelectronics) from the Federal University of Rio de Janeiro in 1989 and 1992, respectively, and his Ph.D. degree (physics-microelectronics) from the University of Grenoble I, France in 1998. From 1992 to 2002 he was a research associate at CERN (European Laboratory for Particle Physics), Geneva, developing solid-state radiation imaging systems for biomedical applications, radiation-hard front-end electronics for high-energy physics detectors and FPGA-based schedulers for 
event-coherent data transfers. Since 2010 he is the holder of the Thales Chair on Advanced Analog Systems at Supélec. His research interests are aerospace, automotive and biomedical systems where the interaction between electronics and the surrounding environment is a fundamental design concern.

Alain Bonnoit was born in 1949. He graduated from Supélec in 1977, where he now is an associate professor. His work focuses on electronic design, CAD of electronic circuits and sensor interface circuits. 